\title{
Periodontal Disease Status in a Population at Najran Province of Saudi Arabia
}

\author{
Ahmed Zafer Alshabab, Monsour Heba Almakrami, Faisal Hassan Almilaq, Ibrahim Saleh Alhareth, \\ Md. Zahid Hossain, Khaled Mashoor Hyderah, Abdulrahman Ahmed Aseri, \\ Sultan Mohammed Alanazi, and Mohammad Abdo Abdulrazzaq
}

\section{ABSTRACT}

Background: The common periodontal diseases are of public health concern, with $20-50$ percent of the global population affected by gingivitis and or periodontitis.

Aim: The aim of this study was to explore the common periodontal diseases occurring among the patients who attended the College of Dentistry hospital of Najran University. And also, to determine the correlation among the age groups, nationalities and risk factors related to periodontal diseases.

Methods: A retrospective hospital-based descriptive type study was performed, providing 352 records of male patients suffering from various types of periodontal diseases. The Centers for Disease Control and the American Academy of Periodontology (CDC-AAP) classification was used for periodontal diagnosis. Patients were grouped by age as group A (6-20 years), group B (21-40 years), group C (41-60 years) and group D (above 60 years). Chi-square test and multinomial logistic regression model were adapted for data analyses.

Results: Out of 352 patients, $217(61 \%)$ and $135(39 \%)$ patients suffered from gingivitis and periodontitis, respectively. Mean age of the patients was $32.81 \pm 15.96$ (range 6-97) years. Sixty-nine $(19.6 \%), 200(56.8 \%), 60$ $(17.0 \%)$ and $23(6.5 \%)$ patients of Group A, B, C and D suffered from different forms of periodontal diseases, respectively. Eighty three percent patients were of Saudis and Yemenis and the rest were of Egyptian, Bangladeshi, Pakistani, Indian, Sudanese, Jordanian, Syrian, Chadian, Ghanaian and Tunisian nationalities. Highest number of patients suffered from moderate generalized gingivitis $142(\mathbf{4 0 . 3 \%})$ and moderate localized periodontitis $57(16.2 \%)$. Majority had plaque \& calculus $290(82.4 \%)$ and the rests had diabetes mellitus, smoking and tobacco chewing habit, asthma, hypertension and heart disease. Correlations among periodontal disease, age group and risk factor were found significant $(p<0.05)$ though between the nationality and risk factor was found not significant ( $p$ 0.999).

Conclusions: Significant relationships were observed among periodontal diseases and age, nationality and risk factors. This small study of Najran may reflect the periodontal status in a population of Saudi Arabia. Further broad scale study would be conclusive establishing such relationship in Saudi Arabian population.

Keywords: Gingivitis, periodontitis, risk factors, correlation, Najran, Saudi Arabia.
Published Online: May 23, 2021

ISSN: 2684-4443

DOI: $10.24018 /$ ejdent.2021.2.3.54

\section{A. Z. Alshabab*}

Assistant Professor, Department of Preventive Dental Sciences, Faculty of Dentistry, Najran University, Saudi Arabia.

(e-mail: azalshabab ${ }^{\circledR}$ nu.edu.sa)

M. H. Almakrami

Intern, Faculty of Dentistry, Najran University, Saudi Arabia.

F. H. Almilaq

Intern, Faculty of Dentistry, Najran University, Saudi Arabia.

I. S. Alhareth

Intern, Faculty of Dentistry, Najran University, Saudi Arabia.

M. Z. Hossain

Associate Professor, Department of Preventive Dental Sciences, Faculty of Dentistry, Najran University, Saudi Arabia.

(e-mail: mzhossain@ ${ }^{\circledR}$ u.edu.sa)

K. M. Hyderah

Assistant Professor, Department of Preventive Dental Sciences, Faculty of Dentistry, Najran University, Saudi Arabia.

(e-mail: kmhydrah@ nu.edu.sa)

A. A. Aseri

Assistant Professor, Department of Preventive Dental Sciences, Faculty of Dentistry, Najran University, Saudi Arabia.

(e-mail: aaaseri@nu.edu.sa)

S. M. Alanazi

Assistant Professor, Department of Preventive Dental Sciences, Faculty of Dentistry, Najran University, Saudi Arabia.

(e-mail: smalanzi@ nu.edu.sa)

M. A. Abdulrazzaq

Lecturer, Department of Preventive

Dental Sciences, Faculty of Dentistry,

Najran University, Saudi Arabia.

*Corresponding Author 


\section{INTRODUCTION}

Common periodontal diseases such as gingivitis and periodontitis are one of the most prevalent oral disorders affecting the human population [1]-[3]. The incidence of periodontal disease is high, with up to $20-50 \%$ of the population worldwide affected by gingivitis or periodontitis [4], [5]. According to recent findings from the Centers for Disease Control and Prevention of USA, half of Americans aged 30 or older have periodontitis, the more advanced form of periodontal disease [5], [6]. High prevalence of periodontal disease in adolescents, adults and older individuals makes it a public health concern [7].

Global Burden of Disease (GBD) study provides information on disease prevalence, incidence, and severity by age, sex, geography, and time. GBD (1990-2015) indicates that the severe periodontal disease affected 538 million people; and total tooth loss affected 276 million people worldwide [8]. Severe periodontal disease was the $11^{\text {th }}$ most prevalent condition in the world based on GBD 2016 [2]. During the last few decades, the burden of periodontal disease has risen significantly, and a wide body of evidence indicates that it is closely associated with systemic diseases [5], [7].

Gingivitis is characterized by red, easily bled edematous gingiva and, if left untreated, the deeper periodontal tissues are implicated in the production of periodontitis that eventually ends in tooth loss [9]. Poor oral hygiene is created by local factors such as bacterial plaque and CALCULUS, which directly irritate periodontal tissues to cause periodontal diseases [10], [11]. Periodontal tissue is also directly affected by smoking and using tobacco products [5], [12], [15]. Systemic conditions and disorders are now considered to be the secondary factors that modulate periodontal disease initiation or progression. Periodontal diseases are evidently linked to common systemic conditions such as diabetes, cardiovascular disease, adverse pregnancy effects, rheumatoid arthritis, chronic obstructive pulmonary disease, chronic kidney disease (CKD), hypertension, multi-organ cancers, etc. [16]-[23]. Local and systemic risk factors play a major predisposing role in the periodontal disease pathogenesis process, influencing the quality of life (QoL) of patients as well [24].

In adults aged 30-40 years, the overall prevalence of periodontitis increases with age and the incidence rises steeply. With the increasing aging population, this burden of periodontitis would continue to increase [25], [26]. While comprehensive studies have been conducted on the prevalence of periodontal diseases in different populations worldwide, data for the Saudi population is poor. Muhammad Nazir et al. [2] suggest that the high prevalence of periodontal disease in the increasingly advancing elderly population justifies the incorporation of programs and policies for the prevention of periodontal disease into general health preventive measures that should start at local, national and international levels.

Prevalence is the number of cases of a disease in a designated population at a given point. This would be an effort of the College of Dentistry at Najran University to conduct a research regarding periodontal status among the dental patients attended the outpatient department since January to December of 2018. Using the research results, a greater effort can be made in providing periodontal health information to the population for raising awareness and to take preventive measures at or around Najran province of Kingdom of Saudi Arabia.

\section{MATERIALS AND METHODS}

\section{A. Examiner Calibration}

Intraoral exams were conducted by qualified and calibrated dental practitioners at the Division of Periodontology, Faculty of Dentistry of Najran University.

\section{B. Study Design}

This research was planned as a retrospective hospitalbased descriptive type study to determine the prevalence of periodontal diseases and potential disease-related risk factors.

\section{Study Population}

A convenience sample was examined of 961 records of patients who visited the College of Dentistry at Najran University's outpatient department (OPD) between January and December 2018. The informed consent was signed by all subjects before their enrollment in the study. Each case was diagnosed as no periodontal disease, gingivitis, and periodontitis and associated risk factors on the basis of periodontal parameters recorded. This study results were obtained from the analysis of 352 samples.

Based on the Canadian Health Measures Survey (20072009), the measurement of loss of periodontal ligament attachment is considered the gold standard in reporting the prevalence of periodontal disease.[27] National Health and Nutrition Examination Survey (NHANES) determined the attachment loss (AL) and probing depth (PD) at six sites of all teeth (excluding third molars) for the estimation of periodontal disease in the U.S. [28].

\section{Study Method}

An observation was made based on:

1. Periodontal diseases prevalence in the different nationalities and age.

2. Prevalence of gingivitis and periodontitis types.

3. Risk factors for periodontal diseases.

4. Association of age, nationality and risk factors with different types of periodontal diseases.

5. Analysis expressed.

The criteria developed by the Centers for Disease Control and the American Academy of Periodontology (CDC-AAP) were followed to identify subjects with periodontitis.[29,30] Periodontal diagnosis criteria: 1) Mild periodontitis- $\geq 3 \mathrm{~mm}$ CAL (clinical attachment loss) in two or more interproximal sites and two or more sites with $\geq 4 \mathrm{~mm}$ PD (probing depth); Or one site with $\mathrm{PD} \geq 5 \mathrm{~mm} ; 2$ ) Moderate periodontitis- $\geq 2$ interproximal sites with $\geq 4 \mathrm{~mm} \mathrm{CAL}$ (not on the same tooth), Or $\geq 2$ sites with $\geq 5 \mathrm{~mm}$ PD (not on the same tooth); and 3) Severe periodontitis- $\geq 2$ interproximal sites with $\geq 6 \mathrm{~mm}$ CAL (not detected on the same tooth) and $\geq 1$ interproximal sites with $\geq 5 \mathrm{~mm}$ PD.

The gingival index (GI) was calculated based on the Ramfjord teeth index [31] as: healthy gingiva, GI score- $<0.1$; mild gingivitis- $\mathrm{GI}=0.1-1$; moderate gingivitis- $\mathrm{GI}=1.1-2$; and severe gingivitis- GI $=2.1-3$. 


\section{E. Statistical Analysis}

Obtained data were coded, entered, and analyzed using the Statistical Package for Social Sciences (SPSS, version 23, IBM Corp, Armonk, NY, USA). Descriptive analysis was done to summarize data as numbers and percentages. Significance testing of differences was done using the chisquared test and t-test to compare data of nominal or interval level, respectively. Multinomial logistic regression analysis was performed to analyze the factors associated with periodontal diseases. All statistical assessments were twotailed and considered significant at $\mathrm{p}<0.05$.

\section{RESULTS:}

Nine hundred and sixty-one patients' records were examined to find out the cases with different types of periodontal diseases. Of them, $352(36.63 \%)$ patients were found sufferers of different types of gingivitis $(217(61 \%))$ and periodontitis $(135(39 \%))$. Mean age of the periodontal diseases sufferers was $32.81 \pm 15.96$ (range 6-97) years.

Table I shows that the Group A (6-20 years) consisted 69 (19.6\%) patients, Group B (21-40 years) 200 (56.8\%), Group C (41-60 years) 60 (17.0\%) and Group D (above 60 years) 23 $(6.5 \%)$ patients.

TABLE I: Distribution OF AgE GROUPS OF PATIENTS SUFFERED FROM

\begin{tabular}{|c|c|c|}
\hline \multicolumn{3}{|c|}{ PERIODONTAL DISEASES } \\
\hline Age group & Frequency & Percent $(\%)$ \\
\hline $\begin{array}{c}\text { Group A } \\
\text { (6-20 Years) }\end{array}$ & 69 & 19.6 \\
\hline $\begin{array}{c}\text { Group B } \\
(21-40 \text { Years })\end{array}$ & 200 & 56.8 \\
\hline $\begin{array}{c}\text { Group C } \\
\text { (41-60 Years) }\end{array}$ & 60 & 17.0 \\
\hline $\begin{array}{c}\text { Group D } \\
\text { (Above } 60 \text { Years) }\end{array}$ & 23 & 6.5 \\
\hline Total & 352 & 100 \\
\hline
\end{tabular}

Table II shows the patients of different nationalities who suffered from periodontal diseases. The Saudi nationals were 164 (46.6\%), Yemeni 128 (36.4\%), Egyptian 16 (4.5\%), Bangladeshi $10(2.8 \%)$, Pakistani $11(3.1 \%)$, Indian $9(2.6 \%)$, Sudanese 7 (2.0\%), Jordanian $2(0.6 \%)$, Syrian $2(0.6 \%)$ patients, and Chadian, Ghanaian and Tunisian patients were only $1(0.3 \%)$ of each nationality.

TABLE II: DiSTRIBUTION OF NATIONALITY OF PATIENTS SUFFERED FROM

\begin{tabular}{ccc} 
& PERIODONTAL DISEASES \\
\hline Nationality & Frequency & Percent $(\%)$ \\
\hline Saudi & 164 & 46.6 \\
Yemeni & 128 & 36.4 \\
Egyptian & 16 & 4.5 \\
Sudanese & 7 & 2.0 \\
Pakistani & 11 & 3.1 \\
Indian & 9 & 2.6 \\
Jordanian & 2 & 0.6 \\
Bangladeshi & 10 & 2.8 \\
Chadian & 1 & 0.3 \\
Syrian & 2 & 0.6 \\
Ghanaian & 1 & 0.3 \\
Tunisian & 1 & 0.3 \\
Total & 352 & 100 \\
\hline
\end{tabular}

In Table III, the total gingivitis cases were found 217 $(61 \%)$. Among those, mild generalized gingivitis cases were $61(17.3 \%)$, moderate generalized gingivitis $142(40.3 \%)$, severe generalized gingivitis $5(1.4 \%)$, puberty gingivitis 2 $(0.6 \%)$ and acute generalized gingivitis were 7 (2.0\%). Total periodontitis cases were $135(39 \%)$. Among those, mild generalized periodontitis cases were $4(1.1 \%)$, moderate generalized periodontitis $45(12.8 \%)$, severe generalized periodontitis $19(5.4 \%)$, mild localized periodontitis 7 (2.0\%), moderate localized periodontitis 57 (16.2\%), severe localized periodontitis $2(0.6 \%)$ and aggressive periodontitis was 1 $(0.3 \%)$.

TABLE III: DISTRIBUTION OF PERIODONTAL DISEASES

\begin{tabular}{ccc}
\hline Periodontal Disease & Frequency & Percent $(\%)$ \\
\hline Mild generalized gingivitis & 61 & 17.3 \\
Moderate generalized gingivitis & 142 & 40.3 \\
Severe generalized gingivitis & 5 & 1.4 \\
generalized puberty gingivitis & 2 & 0.6 \\
Acute generalized gingivitis & 7 & 2.0 \\
Mild generalized periodontitis & 4 & 1.1 \\
Moderate generalized periodontitis & 45 & 12.8 \\
Severe generalized periodontitis & 19 & 5.4 \\
Mild localized periodontitis & 7 & 2.0 \\
Moderate localized periodontitis & 57 & 16.2 \\
Severe localized periodontitis & 2 & 0.6 \\
Aggressive periodontitis & 1 & 0.3 \\
Total & 352 & 100 \\
\hline
\end{tabular}

Out of $352(100 \%)$ patients, plaque \& calculus were found in $290(82.4 \%)$ patients (Table IV). Followed by the patients had diabetes mellitus (DM) 17 (4.8\%), smoking 14 (4.0\%) and tobacco chewing $3(0.9 \%)$ habits, asthma $6(1.7 \%)$, hypertension $6(1.7 \%), \mathrm{DM}+$ hypertension $5(1.4 \%)$, heart disease $3(0.9 \%)$, Asthma + Smoking 2 (0.6\%), DM + asthma, $\mathrm{DM}+$ aspirin, adenoidectomy, deaf and unknown were 1 $(0.3 \%)$ of each risk factors.

TABLE IV: DISTRIBUTION OF RISK FACTORS IN PATIENTS SUFFERED FROM

\begin{tabular}{ccc}
\multicolumn{3}{c}{ PERIODONTAL DISEASES } \\
\hline Risk factors & Frequency & Percent $(\%)$ \\
\hline Plaque \& calculus & 290 & 82.4 \\
Asthma & 6 & 1.7 \\
Smoking & 14 & 4.0 \\
Asthma + Smoking & 2 & 0.6 \\
Adenoidectomy & 1 & 0.3 \\
DM + Aspirin & 1 & 0.3 \\
Anaemia & 1 & 0.3 \\
DM & 17 & 4.8 \\
Hypertension & 6 & 1.7 \\
Heart Disease & 3 & 0.9 \\
Disability & 1 & 0.3 \\
Tobacco chewing & 3 & 0.9 \\
DM + Hypertension & 5 & 1.4 \\
DM + Asthma & 1 & 0.3 \\
Unknown & 1 & 0.3 \\
Total & 352 & 100.0 \\
\hline
\end{tabular}

Table V shows that out of $352(100.0 \%)$ patients, majority $143(39.8 \%)$ suffered from moderate generalized gingivitis, followed by $58(17.1 \%), 57(16.0 \%), 45(12.6 \%), 19(5.3 \%)$, $9(2.0 \%), 7(2.0 \%), 5(1.4 \%), 4(1.1 \%)$ patients suffered from mild generalized gingivitis, moderate localized periodontitis, moderate generalized periodontitis, severe generalized periodontitis, mild localized periodontitis, acute generalized gingivitis, severe generalized gingivitis and mild generalized periodontitis, respectively. Two $(0.60 \%)$ patients of each suffered from Puberty gingivitis and severe localized periodontitis, and only $1(0.30 \%)$ patient suffered from aggressive periodontitis. 
Sixty-nine (19.6\%) patients in Group A (6-20 years) suffered from different forms of gingivitis and periodontitis. Of them, 28 (40.6\%) and 27 (39.1\%) patients suffered from moderate generalized gingivitis and mild generalized gingivitis, respectively. There were 7 (10.1\%), 4 (5.8\%), 2 $(2.9 \%)$ and $1(1.5 \%)$ patient suffered from moderate localized periodontitis, acute generalized gingivitis, puberty gingivitis and mild localized periodontitis, respectively.

In Group B (21-40 years), out of 200 (56.0\%) patients, 97 (48.5\%), 43 (21.5\%) and $27(13.5 \%)$ patients suffered from moderate generalized gingivitis, moderate localized periodontitis and mild generalized gingivitis, respectively. Whereas $12(6.0 \%), 5(2.5 \%), 4$ (2.0\%), 4 (2.0\%), 3 (1.5\%),

and $1(1.5 \%)$ patient suffered from moderate generalized periodontitis, mild localized periodontitis, severe generalized gingivitis, severe generalized periodontitis, acute generalized gingivitis, and aggressive periodontitis, respectively.
Group C (41-60 years) shows that out of $60(17.1 \%)$ patients, $19(31.7 \%)$ patients suffered from moderate generalized periodontitis. Fourteen (23.3\%), 10 (16.7\%), 7 (11.7\%), 4 (6.7\%), 2 (3.3\%), 2 (3.3\%) and 1 (1.7\%) patient suffered from moderate generalized gingivitis, severe generalized periodontitis, moderate localized periodontitis, mild generalized periodontitis, severe localized periodontitis, severe generalized gingivitis and mild localized periodontitis, respectively.

Out of $23(7.0 \%)$ patients in Group D (Above 60 years), 12 $(52.2 \%)$ patients suffered from moderate generalized periodontitis. And $5(21.7 \%), 4(17.4 \%)$ and $2(8.7 \%)$ patients suffered from severe generalized periodontitis, moderate generalized gingivitis and mild generalized periodontitis, respectively.

Correlation between age group and disease diagnoses was found significant $(\mathrm{p}<0.05)$.

TABLE V: CORRELATION BETWEEN AgE GROUP AND PERIODONTAL DisEASES

\begin{tabular}{|c|c|c|c|c|c|c|c|c|c|c|c|c|c|c|c|}
\hline \multirow{15}{*}{ 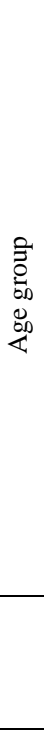 } & \multirow{3}{*}{$\begin{array}{c}\text { Group A } \\
(6-20 \\
\text { Years })\end{array}$} & Count & 27 & 28 & 0 & 2 & 4 & 0 & 0 & 0 & 1 & 7 & 0 & 0 & 69 \\
\hline & & $\begin{array}{c}\text { within } \\
\text { Diagnosis }\end{array}$ & 47.50 & 19.00 & 0.00 & 100.0 & 57.10 & 0.00 & 0.00 & 0.00 & 14.30 & 12.30 & 0.00 & 0.00 & 19.9 \\
\hline & & of Total & 8.10 & 7.60 & 0.00 & 0.60 & 1.10 & 0.00 & 0.00 & 0.00 & 0.30 & 2.00 & 0.00 & 0.00 & 19.9 \\
\hline & \multirow{3}{*}{$\begin{array}{c}\text { Group B } \\
(21-40 \\
\text { Years) }\end{array}$} & Count & 27 & 97 & 4 & 0 & 3 & 0 & 14 & 4 & 7 & 43 & 0 & 1 & 200 \\
\hline & & $\begin{array}{c}\text { within } \\
\text { Diagnosis }\end{array}$ & 44.30 & 68.30 & 80.00 & 0.00 & 42.90 & 0.00 & 26.70 & 21.10 & 71.40 & 75.40 & 0.00 & 100.0 & 56.0 \\
\hline & & of Total & 7.60 & 27.20 & 1.10 & 0.00 & 0.80 & 0.00 & 3.40 & 1.10 & 1.40 & 12.00 & 0.00 & 0.30 & 56.0 \\
\hline & \multirow{3}{*}{$\begin{array}{c}\text { Group C } \\
(41-60 \\
\text { Years) }\end{array}$} & Count & 4 & 14 & 1 & 0 & 0 & 2 & 19 & 10 & 1 & 7 & 2 & 0 & 60 \\
\hline & & $\begin{array}{c}\text { within } \\
\text { Diagnosis }\end{array}$ & 8.20 & 9.90 & 20.00 & 0.00 & 0.00 & 50.00 & 42.20 & 52.60 & 14.30 & 12.30 & 100.0 & 0.00 & 17.10 \\
\hline & & of Total & 1.40 & 3.90 & 0.30 & 0.00 & 0.00 & 0.60 & 5.30 & 2.80 & 0.30 & 2.00 & 0.60 & 0.00 & 17.10 \\
\hline & \multirow{3}{*}{$\begin{array}{c}\text { Group D } \\
\text { (Above } 60 \\
\text { Years) }\end{array}$} & Count & 0 & 4 & 0 & 0 & 0 & 2 & 12 & 5 & 0 & 0 & 0 & 0 & 23 \\
\hline & & $\begin{array}{c}\text { within } \\
\text { Diagnosis }\end{array}$ & 0.00 & 2.80 & 0.00 & 0.00 & 0.00 & 50.00 & 31.10 & 26.30 & 0.00 & 0.00 & 0.00 & 0.00 & 7.00 \\
\hline & & of Total & 0.00 & 1.10 & 0.00 & 0.00 & 0.00 & 0.60 & 3.90 & 1.40 & 0.00 & 0.00 & 0.00 & 0.00 & 7.00 \\
\hline & \multirow{3}{*}{ Total } & Count & 58 & 143 & 5 & 2 & 7 & 4 & 45 & 19 & 9 & 57 & 2 & 1 & 352 \\
\hline & & $\begin{array}{c}\text { within } \\
\text { Diagnosis }\end{array}$ & 100.0 & 100.0 & 100.0 & 100.0 & 100.0 & 100.0 & 100.0 & 100.0 & 100.0 & 100.0 & 100.0 & 100.0 & 100.0 \\
\hline & & of Total & 17.10 & 39.80 & 1.40 & 0.60 & 2.00 & 1.10 & 12.60 & 5.30 & 2.00 & 16.00 & 0.60 & 0.30 & 100.0 \\
\hline
\end{tabular}

Table VI shows that 290 (82.4\%) out of 352 patients who suffered from different forms of periodontal diseases had plaque \& calculus. Seventeen $(4.8 \%)$ patients were suffering from diabetes mellitus (DM), $14(3.9 \%)$ were smokers, 6 $(1.7 \%)$ patients had asthma and $6(1.7 \%)$ patients had hypertension. Five (1.4\%) patients had a combination of DM and hypertension. Rest of the patients [19 (10.1\%)] had heart disease, anaemia, tobacco chewing habit, and so on. Groupwise results found that 63 (91.3\%), $170(85.0 \%), 44(73.3 \%)$ and 8 (34.8\%) patients concerned to groups A, B, C and D, respectively had plaque \& calculus. Plaque \& calculus percentages were found chronologically high to low scales from group A towards D. Whereas, DM and hypertension were found chronologically high to low scales from the higher to lower age groups i.e., groups D towards A. Patients had asthma and DM plus asthma in the relatively younger age groups (A and B). Tobacco chewing habit was also found in groups $\mathrm{B}$ and $\mathrm{C}$. Correlation between age group and risk factors related to periodontal disease was found significant $(\mathrm{p}$ $<0.05$ ).

Patients with various forms of periodontal diseases in Table VII had plaque \& calculus. One hundred and thirty-four (81.7\%) Saudi patients possessed plaque \& calculus out of 164 patients, followed by Egyptians (13 (81.3\%) out of 16),
Yemenis (100 (78.1\%) out of 128), Indians (7 (77.8\%) out of 9) and Bangladeshis (7 (70.0\%) out of 10). All patients $(100 \%)$ of Pakistanis, Sudanese, Jordanians, Syrians, Chadians, Ghanaians, and Tunisians had plaque \& calculus. Diabetic and hypertensive patients were more among the Saudis and Yemenis. Smoking and tobacco chewing habits were observed more in Yemenis and then Saudis. Asthmatic patients were more in Saudis. Minimum quantities were found for other risk factors due to negligible numbers of other nationalities. Correlation between nationality and risk factors related to periodontal disease was found not significant $(\mathrm{p}$ 0.999). 
TABLE VI: CORRELATION BETWEEN AGE GROUPS AND RISK FACTORS

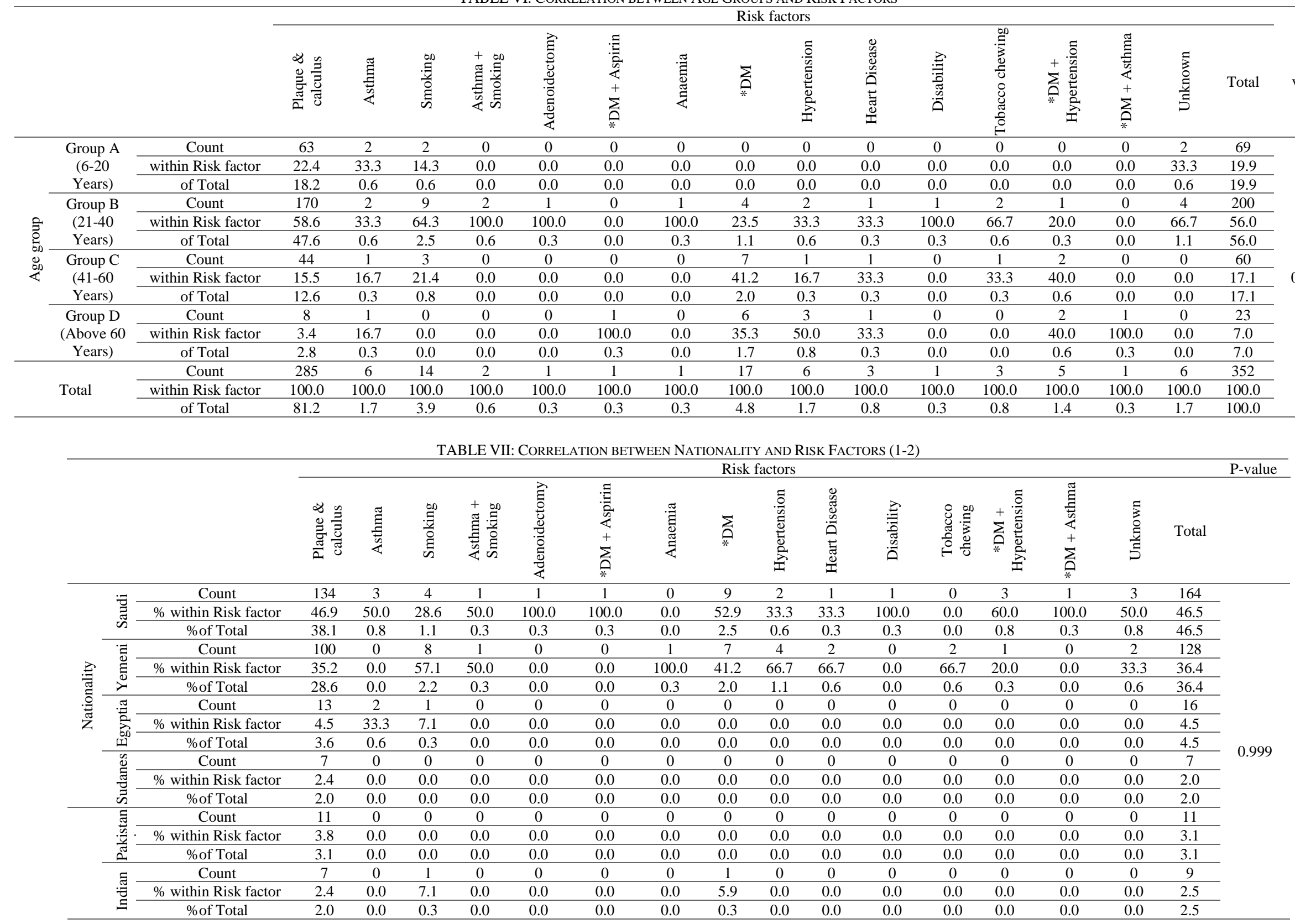


TABLE VII: CORRELATION BETWEEN NATIONALITY AND RISK FACTORS (2-2)

\begin{tabular}{|c|c|c|c|c|c|c|c|c|c|c|c|c|c|c|c|c|c|c|c|}
\hline & & & \multicolumn{16}{|c|}{ Risk factors } & \multirow[b]{2}{*}{ P-value } \\
\hline & & & 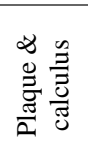 & $\begin{array}{l}\stackrel{\dddot{g}}{\Xi} \\
\text { 萦 }\end{array}$ & 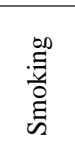 & 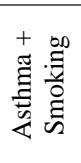 & 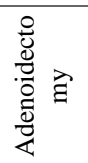 & 苔基 & 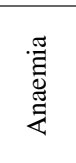 & $\underset{*}{\sum_{*}}$ & 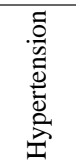 & 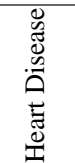 & 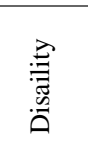 & 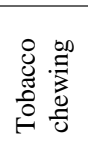 & 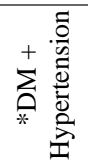 & $\sum_{*}^{+}$ & 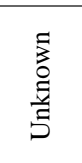 & Total & \\
\hline \multirow{11}{*}{ 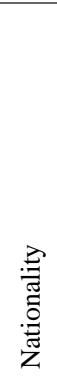 } & \multirow{3}{*}{ Jordanian } & Count & 2 & 0 & 0 & 0 & 0 & 0 & 0 & 0 & 0 & 0 & 0 & 0 & 0 & 0 & 0 & 2 & \multirow{21}{*}{0.999} \\
\hline & & $\%$ within Risk factor & 0.7 & 0.0 & 0.0 & 0.0 & 0.0 & 0.0 & 0.0 & 0.0 & 0.0 & 0.0 & 0.0 & 0.0 & 0.0 & 0.0 & 0.0 & 0.6 & \\
\hline & & $\%$ of Total & 0.6 & 0.0 & 0.0 & 0.0 & 0.0 & 0.0 & 0.0 & 0.0 & 0.0 & 0.0 & 0.0 & 0.0 & 0.0 & 0.0 & 0.0 & 0.6 & \\
\hline & \multirow{3}{*}{ Bangladeshi } & Count & 7 & 1 & 0 & 0 & 0 & 0 & 0 & 0 & 0 & 0 & 0 & 1 & 1 & 0 & 0 & 10 & \\
\hline & & $\%$ within Risk factor & 2.4 & 16.7 & 0.0 & 0.0 & 0.0 & 0.0 & 0.0 & 0.0 & 0.0 & 0.0 & 0.0 & 33.3 & 20.0 & 0.0 & 0.0 & 2.8 & \\
\hline & & $\%$ of Total & 2.0 & 0.3 & 0.0 & 0.0 & 0.0 & 0.0 & 0.0 & 0.0 & 0.0 & 0.0 & 0.0 & 0.3 & 0.3 & 0.0 & 0.0 & 2.8 & \\
\hline & \multirow{3}{*}{ Chadian } & Count & 1 & 0 & 0 & 0 & 0 & 0 & 0 & 0 & 0 & 0 & 0 & 0 & 0 & 0 & 0 & 1 & \\
\hline & & $\%$ within Risk factor & 0.3 & 0.0 & 0.0 & 0.0 & 0.0 & 0.0 & 0.0 & 0.0 & 0.0 & 0.0 & 0.0 & 0.0 & 0.0 & 0.0 & 0.0 & 0.3 & \\
\hline & & \%of Total & 0.3 & 0.0 & 0.0 & 0.0 & 0.0 & 0.0 & 0.0 & 0.0 & 0.0 & 0.0 & 0.0 & 0.0 & 0.0 & 0.0 & 0.0 & 0.3 & \\
\hline & \multirow{3}{*}{ Syrian } & Count & 2 & 0 & 0 & 0 & 0 & 0 & 0 & 0 & 0 & 0 & 0 & 0 & 0 & 0 & 0 & 2 & \\
\hline & & $\%$ within Risk factor & 0.7 & 0.0 & 0.0 & 0.0 & 0.0 & 0.0 & 0.0 & 0.0 & 0.0 & 0.0 & 0.0 & 0.0 & 0.0 & 0.0 & 0.0 & 0.6 & \\
\hline & & $\%$ of Total & 0.6 & 0.0 & 0.0 & 0.0 & 0.0 & 0.0 & 0.0 & 0.0 & 0.0 & 0.0 & 0.0 & 0.0 & 0.0 & 0.0 & 0.0 & 0.6 & \\
\hline & \multirow{3}{*}{ Ghanaian } & Count & 1 & 0 & 0 & 0 & 0 & 0 & 0 & 0 & 0 & 0 & 0 & 0 & 0 & 0 & 0 & 1 & \\
\hline & & $\%$ within Risk factor & 0.3 & 0.0 & 0.0 & 0.0 & 0.0 & 0.0 & 0.0 & 0.0 & 0.0 & 0.0 & 0.0 & 0.0 & 0.0 & 0.0 & 0.0 & 0.3 & \\
\hline & & \%of Total & 0.3 & 0.0 & 0.0 & 0.0 & 0.0 & 0.0 & 0.0 & 0.0 & 0.0 & 0.0 & 0.0 & 0.0 & 0.0 & 0.0 & 0.0 & 0.3 & \\
\hline & \multirow{3}{*}{ Tunisian } & Count & 1 & 0 & 0 & 0 & 0 & 0 & 0 & 0 & 0 & 0 & 0 & 0 & 0 & 0 & 0 & 1 & \\
\hline & & $\%$ within Risk factor & 0.3 & 0.0 & 0.0 & 0.0 & 0.0 & 0.0 & 0.0 & 0.0 & 0.0 & 0.0 & 0.0 & 0.0 & 0.0 & 0.0 & 0.0 & 0.3 & \\
\hline & & $\%$ of Total & 0.3 & 0.0 & 0.0 & 0.0 & 0.0 & 0.0 & 0.0 & 0.0 & 0.0 & 0.0 & 0.0 & 0.0 & 0.0 & 0.0 & 0.0 & 0.3 & \\
\hline & \multirow{3}{*}{ Total } & Count & 286 & 6 & 14 & 2 & 1 & 1 & 1 & 17 & 6 & 3 & 1 & 3 & 5 & 1 & 5 & 352 & \\
\hline & & $\%$ within Risk factor & 100.0 & 100.0 & 100.0 & 100.0 & 100.0 & 100.0 & 100.0 & 100.0 & 100.0 & 100.0 & 100.0 & 100.0 & 100.0 & 100.0 & 100.0 & 100.0 & \\
\hline & & \%of Total & 81.2 & 1.7 & 3.9 & 0.6 & 0.3 & 0.3 & 0.3 & 4.8 & 1.7 & 0.8 & 0.3 & 0.8 & 1.4 & 0.3 & 1.7 & 100.0 & \\
\hline
\end{tabular}


TABLE VIII: CORRELATION BETWEEN NATIONALITY AND PERIODONTAL DiseASE

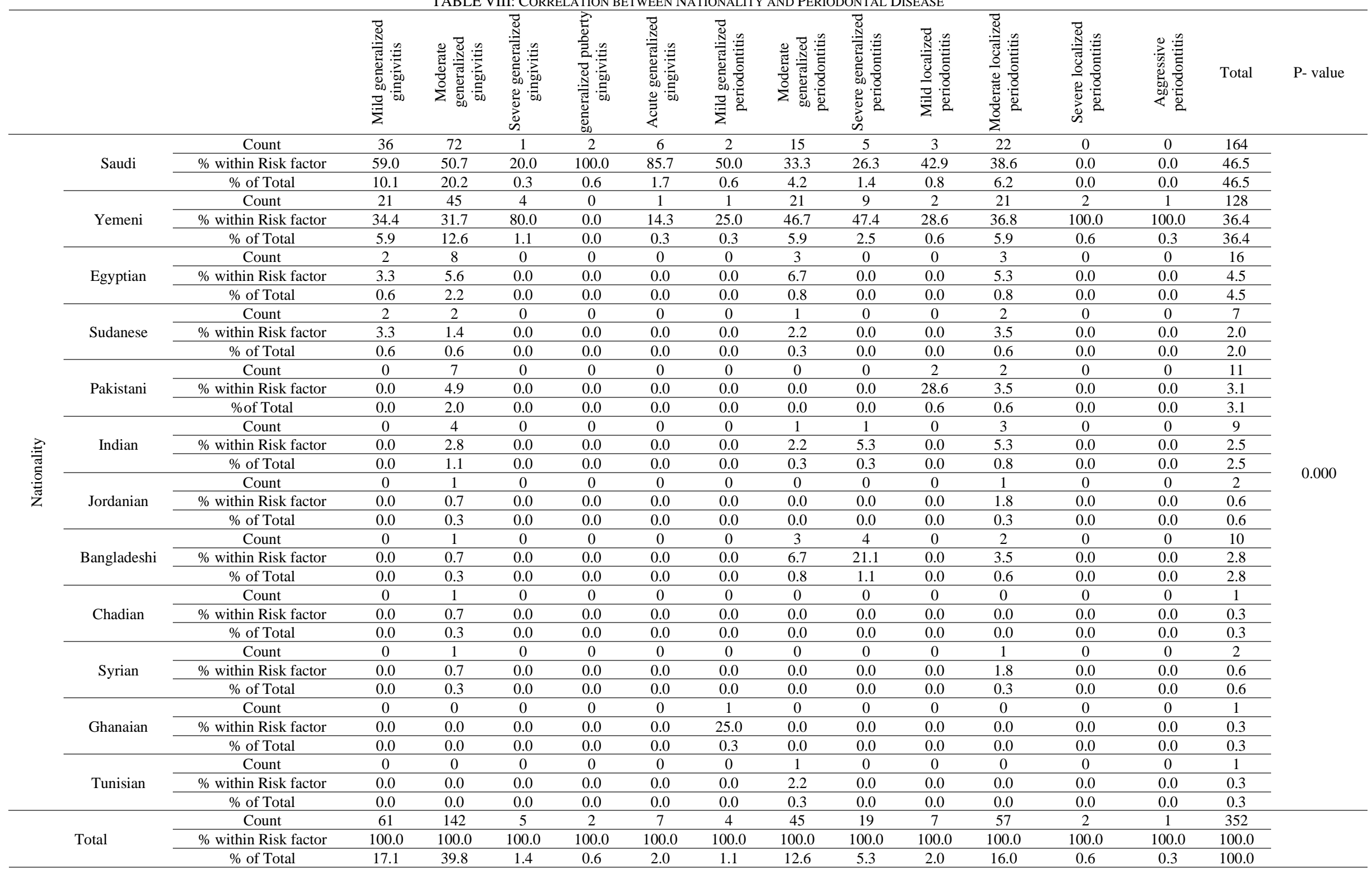


Out of 164 Saudi patients, 72 (43.9\%) suffered from moderate generalized gingivitis (Table VIII). Followed by mild generalized gingivitis $36(22.0 \%)$, moderate localized periodontitis $22(13.4 \%)$, moderate generalized periodontitis $15(9.1 \%)$, acute generalized gingivitis $6(3.7 \%)$, severe generalized periodontitis $5(3.0 \%)$, mild localized periodontitis $3(1.8 \%)$, mild generalized periodontitis 2 $(1.2 \%)$, puberty gingivitis $2(1.2 \%)$ and severe generalized gingivitis $1(0.6 \%)$.

One hundred twenty-eight Yemeni patients suffered from different types of periodontal diseases. Of them, 45 (35.2\%) patients suffered from moderate generalized gingivitis, 21 (16.4\%) patients suffered from each of mild generalized gingivitis, moderate generalized periodontitis and moderate localized periodontitis, $9(7.0 \%)$ severe generalized periodontitis, $4(3.1 \%)$ severe generalized gingivitis, $2(1.6 \%)$ patients suffered from each of mild localized periodontitis and severe localized periodontitis, and $1(0.8 \%)$ patients suffered from each of acute generalized gingivitis, mild generalized periodontitis and aggressive periodontitis. Of the 16 Egyptians, 8 (50.0\%) patients suffered from moderate generalized gingivitis, 3 (18.8\%) patients suffered from each of moderate generalized periodontitis and moderate localized periodontitis and $2(12.5 \%)$ patients suffered from mild generalized gingivitis.

Eleven Pakistanis suffered from moderate generalized gingivitis (7 $(63.6 \%))$, mild localized periodontitis (2 $(18.2 \%)$ ) and moderate localized periodontitis (2 (18.2\%)). Out of 10 Bangladeshis, $4(40.0 \%)$ patients had severe generalized periodontitis, $3(30.0 \%)$ moderate generalized periodontitis, $2(20.0 \%)$ moderate localized periodontitis and $1(10.0 \%)$ patient had moderate generalized gingivitis. Nine Indians suffered from moderate generalized gingivitis (4 $(44.4 \%))$, moderate localized periodontitis $\{3(33.3 \%))$, moderate generalized periodontitis $(1(11.1 \%))$ and severe generalized periodontitis $(1(11.1 \%))$. Out of 7 Sudanese, 2 (28.6\%) patients suffered from each of mild generalized gingivitis, moderate generalized gingivitis and moderate localized periodontitis, and $1(14.3 \%)$ patient suffered from moderate generalized periodontitis. The number of Jordanian, Syrian, Chadian, Ghanaian and Tunisian patients who also suffered from various types of periodontal disease was negligible (Table IX).

Correlation between nationality and periodontal diseases was found significant $(\mathrm{p}<0.05)$.

TABLE IX: EFFECTS OF PERIODONTAL DISEASE ON NATIONALITY, RISK FACTORS AND AGE GROUP

\begin{tabular}{cccc}
\multicolumn{4}{c}{ FACTORS AND AGE GROUP } \\
\hline Model & Chi-Square & df & P-Value \\
\hline Nationality & 43.809 & 144 & 1.000 \\
Risk factor & 122.728 & 168 & 0.997 \\
Age group & 69.130 & 36 & 0.001 \\
\hline
\end{tabular}

Table XI expresses the effects of periodontal disease on nationality, risk factors and age group. The P-value $(1.000$ and 0.997) is >0.05 - Null hypothesis is accepted. Null hypothesis- there is no significant difference (notpredictable) in between dependent variable (periodontal disease) and independent variable (nationality and risk factors). On the other hand, P-value (0.001) is <0.05 - Null hypothesis rejected. Null hypothesis- there is significant difference (predictable) in between dependent variable (periodontal disease) and independent variable (age group).

\section{DISCUSSION}

Gingivitis and periodontitis are widespread periodontal diseases, often with a prevalence that affects 20-50 percent of the world's population. [4], [5] Amare Tefera Biruk Bekele (2020) diagnosed periodontal disease in $42.4 \%$ of Ethiopian patients.[32] The current study found $36.6 \%$ patients suffered from different types of gingivitis $(61 \%)$ and periodontitis $(39 \%)$.

Age can increase vulnerability to the onset and progression of periodontal disease and, thus, age is used as a risk factor in risk assessment tools for preventing the progression of periodontal disease.[33] The aim of the present study was attempted to generate periodontal diseases with agedependent thresholds. Patients were grouped by age as group A (6-20 years), B (21-40 years), C (41-60 years), and group $\mathrm{D}$ (>60 years) in this study. Adolescents with the highest prevalence of no periodontal disease $(21.2 \%)$ compared to adults $(9.3 \%)$ and the older population $(9.7 \%)(\mathrm{P}=0.005)$ were reported by Muhammad Nazir et al., 2020[2]. In the current study, only $19.6 \%$ of group A patients suffered from periodontal diseases (Table V) and the overwhelming majority $(79.7 \%)$ suffered from gingivitis and only $1.5 \%$ of patients had mild localized periodontitis. Group B (21-40 years) consisted $56.0 \%$ patients in this research (Table V). Majority $(62.0 \%)$ of this group suffered from moderate (48.5\%) and mild (13.5\%) generalized gingivitis, and $21.5 \%$ patients suffered from moderate localized periodontitis. In group C (41-60 years), $71.8 \%$ patients suffered from different types of periodontitis and the rest $(28.2 \%)$ suffered from gingivitis. The group $\mathrm{D}$ ( $>60$ years) showed the highest rate $(82.6 \%)$ of periodontitis patients (Table V). Goyal et al.;2019 found that localized chronic periodontitis was the most prevalent in the age group of $25-45$ years $(44.03 \%)$, while generalized chronic periodontitis was observed in the 46-65 age group (12.88\%) [34]. In Hossain MZ et al. [25] study, the prevalence and severity of gingivitis was found increased with age to a peak in the 21-30 years age group, whereas periodontitis increased significantly $(\mathrm{p}<0.001)$ in subjects above the age of 40 years in a population of Abha city of Saudi Arabia. So that, the results of the current study are in accordance with the findings of [2], [34] and [25] studies. It is apparent that the prevalence of periodontal disease has risen with age. Thus, in this study, the association between the age group and periodontal disease was found to be significant $(\mathrm{p}<0.05)$ (Table V).

An evaluation of risk factors should include periodontal diagnosis. Poor oral hygiene combined with plaque and calculus accumulation resulting in gingivitis ultimately leading to periodontitis if left untreated [9]. Periodontal examination was performed on 343 adults attending the outpatient clinics of the Faculty of Dentistry, Cairo University [12]. They found that $58.9 \%$ of participants had calculus deposits. The present study analyzed $82.4 \%$ of patients with plaque \& calculus (Table IV). Kiran Kumar Ganj in the 2017 study observed maximum (53\%) calculus in group I (30-40 years) and minimum (21\%) in group IV (60 years and above) [35]. Group-wise findings in the current study showed that $91.3 \%, 85.0 \%, 73.3 \%$ and $34.8 \%$ patients 
affected by groups A, B, C and D had plaque \& calculus, respectively. The percentages of plaque $\&$ calculus were found chronologically lower than those of higher age groups, i.e., high to low scales from group A to D (Table VI). The risk factors and markers of periodontal disease include smoking, stress, aging, race or ethnicity, and male gender [15]. Smoking and tobacco use habits among male patients of different nationalities with different types of periodontal diseases were identified in this research (Table VI \& VII).

Data shows that periodontal disorders are integrated with systemic diseases and older people suffer more than younger ones.[16]-[23] This research explored the chronologically high to low scales of DM and hypertension from the higher to lower age groups, i.e., groups D to A. The patients in the comparatively younger age groups had asthma and DM plus asthma (A and B) (Table VI \& VII).

Over the last 50 years, research has greatly enhanced our understanding of the biological mechanisms of periodontal diseases. Existing expertise in this area can be further enhanced by scientific collaborations between committed practitioners and comprehensive international older age groups. Significant relationships were observed between periodontal diseases and nationality, age, and risk factors. Future broad scale study would be conclusive establishing such relationship in Saudi Arabian population.

\section{CONCLUSION}

This small study in Najran region may reflect the periodontal status in a population of Saudi Arabia. patients suffered from gingivitis were of younger age groups, whereas patients having periodontitis wee of cooperation on research activities.

\section{DECLARATIONS OF INTEREST}

The authors declare hey do not have conflict of interest.

\section{REFERENCES}

[1] World Health Organization. Periodontal disease. Tech-report series no 207. Geneva: WHO, 1961.

[2] Nazir M, Al-Ansari A, Al-Khalifa K, Alhareky M, Gaffar B, Almas K. Global prevalence of periodontal disease and lack of its surveillance. Scientific World J 2020; 2020: 2146160. Published online 2020 May 28. doi: 10.1155/2020/2146160 PMCID: PMC7275199.

[3] Gross AJ, Paskett KT, Cheever VJ, Lipsky MS. Periodontitis: a global disease and the primary care provider's role. Postgraduate Med J 2017;93(1103):560-65.

[4] Sanz M, D'Aiuto F, Deanfield J, Fernandez-Avilés F. European workshop in periodontal health and cardiovascular disease - scientific evidence on the association between periodontal and cardiovascular diseases: a review of the literature. Euro Heart J Suppl 2010;12 (suppl_B):B3-B12. https://doi.org/10.1093/eurheartj/suq003.

[5] GBD 2017 Disease and Injury Incidence and Prevalence Collaborators. Global, regional, and national incidence, prevalence, and years lived with disability for 328 diseases and injuries for 195 countries, 19902016: a systematic analysis for the Global Burden of Disease Study 2016. Lancet 2017;390(10100):1211-59. doi: 10.1016/S01406736(17)32154-2; PMCID: PMC5605509.

[6] Eke PI, Jaramillo F, Thornton-Evans GO, Borgnakke WS. Dental visits among adult Hispanics-BRFSS 1999 and 2006. J Public Health Dent 2011;71:252-56. PubMed.

[7] Nazir MA. Prevalence of periodontal disease, its association with systemic diseases and prevention. Int J Health Sc 2017;1:72-80.
[8] Kassebaum NJ, Smith AGC, Bernabé E, Fleming TD, Reynolds AE, Vos T, et al. Global, regional, and national prevalence, incidence, and disability-adjusted life years for oral conditions for 195 countries, 1990-2015: a systematic analysis for the global burden of diseases, injuries, and risk factors. J Dent Res 2017;96:380-87.

[9] World Health Organization, Oral Health, World Health Organization, Geneva, Switzerland, 2018. https://www.who.int/news-room/factsheets/detail/oral-health.

[10] Nagpal R, Yamashiro Y, Izumi Y. The two-way association of periodontal infection with systemic disorders: an overview. Mediators Inflamm 2015;2015:793898. doi: 10.1155/2015/793898. Epub 2015 Aug 3. PMID: 26339142; PMCID: PMC4539125.

[11] Șurlin P, Nicolae FM, Șurlin VM, Pătrașcu S, Ungureanu BS, Didilescu AC, Gheonea DI. Could periodontal disease through periopathogen Fusobacterium nucleatum be an aggravating factor for gastric cancer? J Clin Med 2020;9(12): 3885. Published online 2020 Nov 29. doi: 10.3390/jcm9123885 PMCID: PMC7761398 PMID: 33260439.

[12] Abbass MMS, Rady D, Radwan IA, et al. The occurrence of periodontal diseases and its correlation with different risk factors among a convenient sample of adult Egyptian population: a cross$\begin{array}{llll}\text { sectional } & \text { study. } & \text { F1000Research } & \text { 2020;8:1740 }\end{array}$ https://doi.org/10.12688/f1000research.20310.2.

[13] Fons-Badal C, Fons-Font A, Labaig-Rueda C, Fernanda Solá-Ruiz M, Selva-Otaolaurruchi E, Agustín-Panadero R. Analysis of predisposing factors for rapid dental calculus formation. J Clin Med 2020;9(3):858. Published 2020 Mar 20. doi:10.3390/jcm9030858.

[14] Chikte U, Pontes CC, Karangwa I, Kimmie-Dhansay F, Erasmus RT, Kengne AP, Matsha TE. Periodontal disease status among adults from South Africa-prevalence and effect of smoking. Int J Environ Res Public Health 2019;16:3662. doi:10.3390/ijerph16193662.

[15] Gopalasamy K, Kumar PR, Pradeep D. Periodontal status among tobacco users visiting a dental hospital. Int J Pharma Res Jan 2021;13(1):1554-60. https://doi.org/10.31838/ijpr/2021.13.01.232.

[16] Graziani F, Gennai S, Solini A, Petrini M. A systematic review and meta-analysis of epidemiologic observational evidence on the effect of periodontitis on diabetes: an update of the EFP-AAP review. J Clin Periodontol 2018;45(2):167-87.

[17] Nguyen TT, Wu KY, Leclerc M, Pham HM, Tran SD. Cardiovascular diseases and periodontal disease. Current Oral Health Reports 2018;5:13-18. https://doi.org/10.1007/s40496-018-0165-3.

[18] Gil L, Mínguez I, Caffesse R, Llambés FJOHPD. Periodontal disease in pregnancy: the influence of general factors and inflammatory mediators Oral Health Prev Dent 2019;17(1):69-73. doi:10.3290/j.ohpd.a41981.

[19] Li R, Tian C, Postlethwaite A, Jiao Y, Garcia-Godoy F, Pattanaik D, et al. Rheumatoid arthritis and periodontal disease: what are the similarities and differences? Int J Rheumatic Dis 2017;20(12):18871901. https://doi.org/10.1111/1756-185X.13240.

[20] Ferreiraa MKM, de Oliveira Ferreiraa R, Castroa MML, Magnob MB, Almeidaa APCPSC, Fagundesa NCF, Maiab LC, Limaa RR. Is there an association between asthma and periodontal disease among adults? Systematic review and meta-analysis. Life Sciences 2019;223:74-87. https://doi.org/10.1016/j.lfs.2019.03.005.

[21] Zhang J, Jiang H, Sun M, Chen J. Association between periodontal disease and mortality in people with CKD: a meta-analysis of cohort studies. BMC Nephrology 2017;18(1):1-11.

[22] Zhao M-J, Qiao Y-X, Wu L, Huang Q, Li B-H and Zeng X-T. Periodontal disease is associated with increased risk of hypertension: a cross-sectional study. Front Physiol 2019;10:440. doi: 10.3389/fphys.2019.00440.

[23] Viola T. Study shows link between periodontal disease and increased risk of colorectal cancer. Cancer Prev Res 2020;13(8):699-706.

[24] Surlin P, Gheorghe D-N, opescu D, Lazar L, Luchian I, Martu M-A. Multiple local and systemic conditions as predisposing risk factors for periodontal disease evolution and decreased quality of life. Romanian J Med Dent Edu 2020;9(2):26-30.

[25] Hossain MZ, Fageeh HN, Elagib MFA. Prevalence of periodontal diseases among patients attending the outpatient department at the College of Dentistry, King Khalid University, Abha, Saudi Arabia. City $\begin{array}{llll}\text { Dent Coll J 2013;10(1):9-12 } & \end{array}$ https://doi.org/10.3329/cdcj.v10i1.13835

[26] Tonetti MS, Bottenberg P, Conrads G, Eickholz P, Heasman P, et al Dental caries and periodontal diseases in the ageing population: call to action to protect and enhance oral health and well- being as an essential component of healthy ageing - Consensus report of group 4 of the joint EFP/ORCA workshop on the boundaries between caries and periodontal diseases. J ClinPeriodontol 2017;44(suppl 18):S135-S144. 
[27] Health Canada. Report on the Findings of the Oral Health Component of the Canadian Health Measures Survey; 2007-2009. [http://www.fptdwg.ca/assets/PDF/CHMS/CHMS-E-summ.pdf.

[28] Eke PI, Dye BA, Wei L, Thornton-Evans GO, Genco RJ. CDC periodontal disease surveillance workgroup: James Beck (University of North Carolina, Chapel Hill, USA), et al. Prevalence of periodontitis in adults in the United States: 2009 and 2010. J Dent Res 2012;91:91420. [doi:10.1177/0022034512457373.

[29] Page RC, Eke PI. Case definitions for use in population-based surveillance of periodontitis. J Periodontol 2007;78(7):1387-99.

[30] Eke PI, Page RC, Wei L, Thornton-Evans G, Genco RJ. Update of the case definitions for population-based surveillance of periodontitis. J Periodontol 2012;83(12):1449-54.

[31] Löe H. The Gingival Index, the Plaque Index and the Retention Index Systems. J Periodontol 1967;38(Suppl 6):610-16.

[32] Bekele ATB. Periodontal disease status and associated risk factors in patients attending a tertiary hospital in northwest Ethiopia. Clin Cosmetic Investigat Dent 2020;12:485-92.

[33] Lang NP, Suvan JE, Tonetti MS. Risk factor assessment tools for the prevention of periodontitis progression a systematic review. J Clin Periodontol 2015;42(Suppl 16): $\quad$ S59-S70. https://doi.org/10.1111/jcpe.12350.

[34] Goyal J, Menon I, Singh RP, Gupta R, Sharma A, Bhagia P. Prevalence of periodontal status among nicotine dependent individuals of 35-44 years attending community dental camps in Ghaziabad district, Uttar Pradesh. J Family Med Prim Care 2019;8:2456-62.

[35] Kiran Kumar Ganj. Prevalence of chronic periodontitis and its associated risk indicators among Saudi nationals in Aljouf province Kingdom of Saudi Arabia. Saudi J Oral Dent Res 2017;2(9):230-35. DOI:10.21276/sjodr.2017.2.9.1

[36] S. Chen, B. Mulgrew, and P. M. Grant, "A clustering technique for digital communications channel equalization using radial basis function networks," IEEE Trans. on Neural Networks, vol. 4, pp. 570578, July 1993.

[37] J. U. Duncombe, "Infrared navigation-Part I: An assessment of feasibility," IEEE Trans. Electron Devices, vol. ED-11, pp. 34-39, Jan. 1959.

[38] C. Y. Lin, M. Wu, J. A. Bloom, I. J. Cox, and M. Miller, "Rotation, scale, and translation resilient public watermarking for images," IEEE Trans. Image Process., vol. 10, no. 5, pp. 767-782, May 2001. 